\title{
Something may change in the management of uncomplicated acute appendicitis
}

Seven percent of humans will experience acute appendicitis (AA) in their lifetime. ${ }^{1}$ For the past 130 years, appendectomy has been the most common emergency surgery. ${ }^{1-2}$ Only in the case of appendiceal adhesions or masses, antibiotic treatment is preferred over immediate surgery.

In uncomplicated acute appendicitis (UAA), inflammation is limited to the appendix, while the appendiceal wall maintains integrity. Patients are in good shape and have the typical signs and pain in the right iliac fossa. An ultrasound shows that the appendix is non-compressible and measures more than $6 \mathrm{~mm}$ in diameter. Mesenteric fat is hyperechoic and little clear liquid may be observed in the periappendiceal or retrovesical areas. In complicated AA, patients' general health status is impaired and there is evidence of perforation, periappendiceal abscess or generalized peritonitis. There is no discussion regarding surgery in complicated AA.

Antibiotic treatment for UAA has been proposed in the past decade to reduce the costs of the high number of appendectomies and prevent surgical stress and complications of general anesthesia, which occurred in 7-17 \% of cases. ${ }^{1-4}$ Case series meta-analyses have been published, which included adults with UAA treated exclusively with antibiotics, and results have been dissimilar: antibiotics were effective in 68-95 \% of cases and the rate of AA recurrence ranged from $4.4 \%$ to $39 \% .^{3-6}$ In a 2016 meta-analysis of five series that included 1430 adults with UAA, antibiotic treatment was effective in $63 \%$. However, $53-79 \%$ of patients underwent surgery in the end. These studies have a low quality of evidence in terms of therapeutic outcomes due to their design defects. ${ }^{7}$

In 2017, a meta-analysis selected 10 studies that investigated antibiotic treatment and surgery for UAA outcomes in 413 children. Antibiotic treatment was effective in $97 \%$ of patients, and surgery was prevented in $82 \%$. Both complications and the length of stay were similar. AA recurrence after non-surgical treatment was $14 \% .{ }^{1}$ Only six of these studies were comparative, and only one was randomized, although the number of cases was small. ${ }^{2}$ This pilot study showed that, with antibiotic treatment given for UAA, the rate of complications and AA recurrence was acceptable and that late appendectomy due to drug treatment failure did not result in more complications than immediate surgery. $2,4,6$

Up to 2018, no study in children has been conducted that showed sufficient statistical power to state that antibiotic treatment for UAA is superior to surgery. $1,4,6$

In 2017, a multicenter, randomized study was started which, in accordance with statistical protocols and strict ethical aspects, will compare the results of antibiotic treatment and immediate surgery for UAA. It will compare the outcomes of antibiotic treatment and immediate surgery for UAA. This study, known as the APPY trial, plans to recruit 978 children with UAA aged 5-16 years with follow-up for 1 year after the initial event. ${ }^{6}$ UAA diagnosis will be made based on clinical criteria and ultrasound, and a computed tomography (CT) with oral contrast administration will be used only if clinical and/ or ultrasound diagnosis is unclear. Patients with suspicion of perforated appendicitis or who received more than one round of antibiotics or younger than 5 years will be excluded. In young children, AA progresses rapidly, it may present atypically and, in 12.5-30\% of cases, an initial appendiceal perforation is observed..$^{6,89}$ Children with a history of other events compatible with AA, cystic fibrosis or malignancies, and pregnant adolescents will be also excluded. The presence of an appendiceal fecalith will not exclude a child from study participation. ${ }^{6}$ Some authors advise against non-surgical treatment in these cases, due to the higher incidence of complications. ${ }^{2-4}$

Eligible children with UAA will be randomly assigned to the antibiotic treatment selected by each site or to immediate videolaparoscopic surgery as the best surgical option. ${ }^{6}$ Antibiotics will be administered intravenously for at least 12 hours and outcomes will be assessed at 24 and 48 hours. If an adequate response is observed, children will complete 10 days of antibiotics given orally. If the patient's status worsens within 24 hours or no improvement is observed within 48 hours of initiation, surgery will be indicated.

The consent for the randomization of children with UAA will be obtained. For the pilot study of the APPY trial, only $40 \%$ of the parents of eligible children gave their consent for randomization. ${ }^{2}$ Another study in children with 
UAA could not be carried out in a randomized manner because, although it reported a $98.7 \%$ effectiveness with antibiotic treatment, it did not show statistical power. ${ }^{3}$

If the APPY trial showed that antibiotic treatment for UAA is better than immediate surgery, it would create a revolution in the field of pediatric surgery.

\section{This deserves some considerations:}

A pediatric surgeon would diagnose UAA, a common condition in their daily practice, and would only perform a surgery in case of antibiotic treatment failure. ${ }^{4}$ If the diagnosis of UAA and the non-surgical therapeutic decision were to be done exclusively by a clinical pediatrician, it would be controversial. Many times, AA diagnosis is challenging, and delays occur in 27-57 \% of cases. At facilities with a higher level of care, $4-6 \%$ of appendectomies were done in patients with a healthy appendix. ${ }^{6,9}$

In $\mathrm{AA}$, an ultrasound has a sensitivity of $99 \%$ and a specificity of $95 \%$. Only in doubtful cases, a CT scan with oral contrast administration would be used, but this test is not infallible and may overlook a perforation.

If children with an "alleged" UAA do not undergo surgery, antibiotic treatment would be unnecessary if they had viral mesenteric adenitis or ruptured ovarian follicles. In addition, children with an infected or complicated Meckel's diverticulum or other surgical causes of intraperitoneal infection who do not undergo surgery in a timely manner would be put at risk.

AA is a common reason for medical malpractice litigation. Both a non-surgical conduct in UAA and a 48-hour surgery delay when antibiotic treatment is ineffective require validation.

Medicine practiced "on the defensive" results in unnecessary studies. Not performing an ultrasound or CT scan, which would provide a more certain diagnosis of UAA, may cause the inappropriate referral of a patient with a low complexity condition to tertiary care facilities.

If, in our setting, we are looking to reduce the costs of drug treatment for UAA, the length of stay in such cases should be similar to that of a videolaparoscopic appendectomy. A nonrandomized study carried out in children with UAA showed a longer average length of stay of 5 days for antibiotic treatment. ${ }^{8}$ In another Japanese study, the length of stay for drug treatment in UAA was 6 days based on health care system preferences. ${ }^{3}$ In each case, the decision should be made based on the family's level of alarm so as to complete an outpatient treatment.

Not having to undergo surgery in the case of an UAA appeals both children and parents, but there is uncertainty regarding the effectiveness of drug treatment. In a study, 82 out of 197 patients with UAA did not respond to the antibiotics and underwent surgery (42\%). ${ }^{8}$ Once the child overcomes the initial event, the fear of recurrence will linger, especially in the case of patients living in rural areas or traveling for vacations, education, camping, sporting tours, etc. A study showed UAA recurrence in $28 \%$ of patients who received antibiotics. ${ }^{3}$ Publications showed that more than $40 \%$ of children with UAA treated with antibiotics were readmitted to the hospital and finally underwent surgery. ${ }^{8,10}$

Statistical proof is required to demonstrate that drug treatment for UAA in children older than 5 years is more effective than immediate surgery, has fewer complications and a low level of recurrence. Besides, patient selection criteria should be defined. ${ }^{6}$

Until outcomes support the selection of drug treatment for UAA, the current indication for early surgery should not be modified. ${ }^{2,4,6}$

Fernando L. Heinen, MD Specialist in Pediatric Surgery Sanatorio de Los Arcos (SMG) Autonomous City of Buenos Aires fernando.heinen@gmail.com

http: / / dx.doi.org/ 10.5546/aap.2019.eng.68

To cite: Heinen FL. Something may change in the management of uncomplicated acute appendicitis. Arch Argent Pediatr2017; 117(2):68-70.

\section{REFERENCES}

1. Georgiou R, EatonS, Stanton MP, Pierro A, etal. Efficacy and Safety of Nonoperative Treatment for Acute Appendicitis: A Meta-analysis. Pediatrics. 2017; 139(3):e20163003.

2. Svensson JF, Patkova B, Almstrom M, Naji H, et al. Nonoperative treatment with antibiotics versus surgery for acute nonperforated appendicitis in children: a pilot randomized controlled trial. Ann Surg. 2015; 261(1):67-7.1

3. Tanaka $\mathrm{Y}$, Uchida H, Kawashima H, Fujiogi M, et al. Long-term outcomes of operative versus nonoperative treatment for uncomplicated appendicitis. J Pediatr Surg. 2015; 50(11):1893-7.

4. Gorter R, Van der Lee JH, Heijsters FA, Cense HA, et al. Outcome of initially nonoperative treatment for acute simple appendicitis in children. J Pediatr Surg. 2018; 53(9):1849-54. 
5. Mason RJ, Moazzez A, Sohn H, Katkhouda N. Metaanalysis of randomized trials comparing antibiotic therapy with appendectomy for acute uncomplicated (no abscess or phlegmon) appendicitis. Surg Infect (Larchmt). 2012; 13(2):74-84.

6. Hall NJ, EatonS, Abbo O, Arnaud AP, et al. Appendectomy versus non-operative treatment for acute uncomplicated appendicitis in children: study protocol for a multicentre, open-label, non-inferiority, randomised controlled trial. BMJ Paediatr Open. 2017; 1(1):e000028.

7. Rollins KE, Varadhan KK, Neal KR, Lobo DN. Antibiotics versus appendicectomy for the treatment of uncomplicated acute appendicitis: an updated meta-analysis of randomized controlled trials. World J Surg. 2016; 40(10):2305-18.

8. Caruso AM, Pane A, Garau R, Atzori P, et al. Acute appendicitis in children: not only surgical treatment. $J$ Pediatr Surg. 2017; 52(3):444-8.

9. Marzuillo P, Germani C, Krauss BS, Barbi E. Appendicitis in children less than five years old: a challenge for the general practitioner. World J Clin Pediatr. 2015; 4(2):19-24.

10. Bachur RG, Lipsett SC, Monuteaux MC. Outcomes of Nonoperative Management of Uncomplicated Appendicitis. Pediatrics. 2017; 140(1):e20170048.

\section{Pacifiers: Counseling instead of prohibiting. The new Baby- friendly Hospital Initiative}

\section{The beginning}

The first few hours and days of a newborn's life are a critical window for establishing lactation and for providing mothers with the support they need to breastfeed successfully. Breast milk is considered the biological norm to feed the baby and a preventive action for both the mother (cancer) and the infant (infections).

The joint World Health Organization (WHO)/ United Nations International Children's Emergency Fund (UNICEF) statement (Geneva, 1989) urged all mother and child health care services to implement the measures summarized in the "Ten Steps to Successful Breastfeeding."1

One year later, the "Innocenti Declaration" was launched after a meeting held at the Ospedale degli Innocenti, Florence, Italy. The declaration made a strong appeal to governments worldwide to support breastfeeding through programs and legislation, such as breastfeeding rights of working women, and prioritized the implementation of the "Ten Steps to Successful Breastfeeding." ${ }^{2}$

\section{Baby-friendly Hospital Initiative}

Both statements may be considered the immediate precedent of the Baby-friendly Hospital Initiative (BFHI). The BFHI establishes that the Ten Steps to Successful Breastfeeding are mandatory at the hospitals certified as babyfriendly by the UNICEF.

It is estimated that, at present, only $10 \%$ of births worldwide take place at facilities that have been designated as baby-friendly hospitals according to the BFHI. ${ }^{3}$ Although it has been demonstrated that the program promotes breastfeeding among mothers with a low level of education, ${ }^{4}$ it has met with criticism in terms of its difficult sustainability. The vertical enforcement of recommendations has also been questioned. These considerations were outlined in the Executive Summary of the new BFHI implementation guidance. ${ }^{3}$

I personally believe that the original Step 9 "Give no artificial teats or pacifiers (also called dummies or soothers) to breastfeeding infants" was worded this way due to the prevalence of fundamentalism over science, which had been adopted by certain professional associations that had managed to institutionalize a strict and unyielding behavior in relation to breastfeeding that took form throughout child care settings.

Such stance was sustained at a time when the percentage of exclusively breastfed infants was really small, which resulted in a high infant morbidity and mortality (gut microbiota alteration and milk contamination). This way, breastfeeding advocacy became a life-saving intervention.

The recommendation against the use of pacifiers was based on alleged risk factors that were not confirmed in randomized controlled trials, which is the best evidence to assess a health intervention. ${ }^{5}$ Step 9, as it was written in 1989, constitutes the unethical dogma of breastfeeding ${ }^{6}$ because it has been demonstrated that pacifiers during the baby's sleep time reduce the risk for sudden infant death syndrome. ${ }^{7}$ At the time the Ten Steps were developed, there was no epidemiological evidence showing that pacifier use was harmful for breastfeeding. It is worth noting that Victora et al. published the 